\section{Identifying Risk Factors Associated with Intrapartum Stillbirth and Neonatal Mortality in Rural Ghana}

\author{
Megan P Donohue ${ }^{1}$, Jessica L Top ${ }^{1}$ and Colleen A Kraft ${ }^{2}$ \\ ${ }^{1}$ Virginia Tech Carilion School of Medicine, Roanoke, VA, USA \\ ${ }^{2}$ Cincinnati Children's Hospital Medical Center, Cincinnati, OH, USA
}

\begin{abstract}
Background: Under-5 mortality in Sub-Saharan Africa has decreased over the past two decades, yet neonatal morality remains high. A lack of reliable data is available in low-resource countries to inform interventions to reduce neonatal mortality. Local data from community hospitals can provide information and characteristics that may identify risk factors associated with neonatal mortality and stillbirth. This clinic-based data can be used to predict survival and inform community level interventions.
\end{abstract}

Materials and methods: Retrospective chart review collected data on all deliveries between the period of October 2011 and February 2013 at a single hospital in rural Ghana. Maternal characteristics, neonatal characteristics and delivery narratives were collected. Variables collected included maternal age, previous number of pregnancies and previous number of deliveries. Neonatal characteristics recorded included gestational age, birth weight, Apgar scores and survival. Descriptive characteristics of the all deliveries were stratified by neonatal survival. Studentized t-tests, Wilcoxon rank sum and chi-squared analysis were used detect differences between those that survived and those that died. Univariate logistic regression analysis was then used to assess odds of neonatal mortality based on gravida and parity. In addition, chart narratives of neonatal deaths were used to identify most common causes of death.

Results: Data from 1239 deliveries was reviewed, including 54 fetal deaths within 48 hours after delivery. Increased number of previous pregnancies $(p=0.0270)$, five or more previous deliveries $(p=0.0460)$ and decreased birth weight $(p=0.0089)$ were all associated with fetal demise. Furthermore, categorical univariate logistic regression

*Corresponding author: Colleen A Kraft, Cincinnati Children's Hospita Medical Center, 3333 Burnet Ave, MLC 15018, Cincinnati, OH 45229, USA, Tel: +1 5138036806; Fax: +1 8664156779; E-mail: Colleen.Kraft@cchmc.org

Citation: Donohue MP, Top JL, Kraft CA (2015) Identifying Risk Factors Associated with Intrapartum Stillbirth and Neonatal Mortality in Rural Ghana. J Neonatol Clin Pediatr 2: 010.

Received: February 23, 2015; Accepted: April 27, 2015; Published: May 12, 2015 analysis found that women with five or more previous deliveries were 2.24 times as likely to have delivery result in fetal demise than those with less than five previous deliveries $(p=0.0350)$. Preliminary analysis suggests most deaths were due to complications encountered during labor.

Conclusion: Neonatal mortality is drawing increased attention by the World Health Organization (WHO) and United Nations (UN) at this time. In one rural hospital, neonatal death within the first 48 hours was associated with increased gravida and parity and decreased birth weight. These findings should be used to develop risk screening tools that can be used at the community level.

Keywords: Ghana, Local data, Neonatal, Rural

\section{Introduction}

Between 1990 and 2015, worldwide progress has been made toward achieving the United Nation's Millennium Development Goal of reducing under-five mortality by two-thirds to reach a global target of 32 deaths/1000 live births [1]. However, deaths within the first 28 days of life, the neonatal period, have not dropped accordingly. Neonatal mortality remains a vital area of focus in order to reach the Millennium Development Goals. In, 2012, Sub-Saharan Africa reported the highest risk of neonatal mortality worldwide (32 deaths/1000 live births). Ghana's neonatal mortality rate in 2012 was reported as 28 deaths/1,000 live births [2] with a stillbirth rate of $24 / 1000$. This compares to a rate of 4 neonatal deaths/1000 live births in the developed world [3]. The majority of the world's stillbirths and neonatal deaths occur where no clinical information is available to guide programs, and Ghana is no exception.

Much of the data used to estimate perinatal mortality in many West African countries are derived from complex statistical modeling techniques or from nationally representative demographic and health surveys. Despite the high neonatal mortality rate in Sub-Saharan Africa, individual data identifying risk factors for neonatal mortality and intrapartum stillbirth from the region remains sparse [4]. For this reason, infant mortality remains highest in the rural and central regions of Ghana, including Kasei where this study takes place [5]. This project seeks to identify risk factors of death within $48 \mathrm{hr}$ of delivery as a proximate measure of neonatal death. This information may then be used to guide local interventions.

\section{Methods}

St. Luke Hospital is a small, private hospital in Kasei, Ghana which serves an estimated catchment area of 300,000 individuals. The hospital has 24 adult inpatient beds, 8 pediatric inpatient beds, 4 surgical suites and a recently added 8 bed labor and delivery ward. Kasei is a rural village on the northern outskirts of the Ashanti region of central Ghana. The majority of inhabitants in this district are subsistence farmers who live in small, scattered villages. Patient records are kept in handwritten log books and charts.

Retrospective chart review on all deliveries at St. Luke's Hospital in Kasei, Ghana between the period of October 2011 and February 2013 was performed. The primary objective was to assess the rate of morality within $48 \mathrm{hrs}$ of delivery and to identify associated risk factors. 
To assess rate of neonatal mortality, delivery information was collected from local log books and entered into Microsoft access database for analysis. Variables included in the review were divided into maternal and fetal/neonatal. Maternal characteristics collected included age, number of total pregnancies, number of previous deliveries, gestational age at delivery and initial delivery outcome (stillbirth, live birth). Fetal/neonatal characteristics collected included gender, birth weight, Apgar scores at 1 and 5 minutes and neonatal mortality.

Data was analyzed using SAS Version 9.2. Descriptive analyses were conducted to describe maternal age, gestational age, birth weight, and gender. Mean was reported for variables with normal distribution including maternal age, and birth weight. Median was reported for variables with non-normal distribution including number of previous pregnancies, number of previous deliveries and gestational age.

All results were stratified by survival. Studentized t-tests were used to calculate $\mathrm{p}$-values for normally distributed variables. Wilcoxon Rank Sum was used to calculate p-values for variables of skewed distribution. Chi-square tests were performed to calculate p-values for categorical variables. Number of previous maternal pregnancies and number of previous maternal deliveries were analyzed as both as continuous variables and categorical variables in order to further elucidate the relationship of these risk factors with neonatal mortality.

Univariate logistic regression analysis was performed to further assess the relationship between number of previous pregnancies, number of previous deliveries and neonatal mortality. Number of previous pregnancies and number of previous deliveries were each examined as categorical independent variables for this analysis. Odds ratios with $95 \%$ confidence intervals were computed. Prior to analysis a p-value $<0.05$ was set for significance.

All methods were approved by Virginia Tech Institutional Review Board.

\section{Results}

Data from 1239 deliveries and 1186 mothers between October 2011 and February 2013 were included in this analysis. Fifty-four (4.35\%) deliveries resulted in a fetal death within the first 48 hours of life. This is a rate of 45 deaths/1000 live births. For all deliveries, the mean maternal age was 26.1 years. The median number of previous maternal pregnancies was 2 and median number of previous maternal deliveries was 1 . Median gestational age was 38 weeks. Mean birth weight was 2.9 kilograms. When stratified by neonatal survival, there was a statistically significant difference between groups for number of previous maternal pregnancies (gravida) when examined as a continuous variable $(\mathrm{p}=0.0270)$. However, when analyzed as a categorical variable of less than 5 pregnancies vs 5 and greater pregnancies, no significant difference was found between groups $(\mathrm{p}=0.1048)$. Number of previous maternal deliveries (para) did not significantly different between survival strata when examined as a continuous variable $(p=0.0512)$, but was significantly different between strata as a categorical value less than 5 vs 5 and greater $(\mathrm{p}=0.0460)$. Birth weight also differed significantly $(\mathrm{p}=0.0089)$. Maternal age $(\mathrm{p}=0.0792)$ and gestational age $(\mathrm{p}=0.9083)$ were not statistically different between neonates that survived and those that died. Results are shown in table 1.

Categorical univariate logistic regression analysis was performed to further elucidate the relationship between number of maternal

\begin{tabular}{|c|c|c|c|c|}
\hline & $\begin{array}{c}\text { All } \\
\text { Deliveries }\end{array}$ & $\begin{array}{c}\text { Fetal } \\
\text { Survival }\end{array}$ & $\begin{array}{c}\text { Fetal } \\
\text { Demise }\end{array}$ & p-value \\
\hline $\begin{array}{l}\text { Maternal Age--mean } \\
\text { (Range) }\end{array}$ & $\begin{array}{c}26.1 \\
(13-50)\end{array}$ & $\begin{array}{c}26.0 \\
(14-50)\end{array}$ & $\begin{array}{c}27.6 \\
(13-42)\end{array}$ & 0.0792 \\
\hline $\begin{array}{c}\text { Maternal Number of Previ- } \\
\text { ous Pregnancies--median } \\
\text { (Range) }\end{array}$ & $\begin{array}{c}2 \\
(1-13)\end{array}$ & $\begin{array}{c}2 \\
(1-13)\end{array}$ & $\begin{array}{c}3 \\
(1-9)\end{array}$ & $0.0270^{*}$ \\
\hline Less than 5 vs $5+$ & 234 & 219 & 15 & 0.1048 \\
\hline $\begin{array}{l}\text { Maternal Number of Previ- } \\
\text { ous Deliveries--median }\end{array}$ & $\begin{array}{c}1 \\
(0-9) \\
\end{array}$ & $\begin{array}{c}1 \\
(0-9) \\
\end{array}$ & $\begin{array}{c}1 \\
(0-7) \\
\end{array}$ & 0.0512 \\
\hline Less than 5 vs $5^{+}$ & 109 & 100 & 9 & $0.0460^{*}$ \\
\hline $\begin{array}{l}\text { Gestational Age } \\
\text { (Range) }\end{array}$ & $\begin{array}{c}38 \\
(21-43)\end{array}$ & $\begin{array}{c}38 \\
(21-43)\end{array}$ & $\begin{array}{c}38 \\
(23-42)\end{array}$ & 0.9083 \\
\hline $\begin{array}{l}\text { Birth weight } \mathrm{Kg} \\
\quad \text { (Range) }\end{array}$ & $\begin{array}{c}2.9 \\
(1.0-4.6)\end{array}$ & $\begin{array}{c}2.9 \\
(1.0-4.6) \\
\end{array}$ & $\begin{array}{c}2.6 \\
(1.3-4.2)\end{array}$ & $0.0089^{*}$ \\
\hline
\end{tabular}

Table 1: Descriptive characteristics of fetus stratified by survival. pregnancies, number of maternal deliveries with neonatal death. Findings are reported in table 2.

\begin{tabular}{|c|c|c|c|}
\hline & Odds Ratio & $95 \%$ Cl & P-value \\
\hline \multicolumn{4}{|c|}{ Number of Previous Maternal Pregnancies } \\
\hline$>/=4$ & 1.6036 & 0.9085 to 2.8305 & 0.1033 \\
\hline$>/=5$ & 1.6068 & 0.8519 to 3.3037 & 0.1430 \\
\hline \multicolumn{4}{|c|}{ Number of Previous Maternal Deliveries } \\
\hline$>/=4$ & 1.7231 & 0.8989 to 3.3033 & 0.1013 \\
\hline$>/=5$ & 2.2436 & 1.0585 to 4.7552 & $0.0350^{*}$ \\
\hline
\end{tabular}

Table 2: Categorical univariate logistic regression analysis.

Number of previous maternal pregnancies was defined as a categorical variable to compare mothers with less than four previous pregnancies to those with four or more previous pregnancies. The odds ratio for mothers with four or more previous pregnancies was 1.6036 with a $95 \%$ confidence interval of 0.9085 to 2.8305 and a $\mathrm{p}$-value of 0.1033 . The same variable was again modeled to compare mothers with less than five previous pregnancies to those with five or more previous pregnancies. The odds ratio for mothers with five or more previous pregnancies was 1.6068 with a $95 \%$ confidence interval of 0.8519 to 3.037 with a p-value of 0.1430 .

Number of previous maternal deliveries was also modeled categorically to predict neonatal mortality. The odds ratio for mothers with four or more previous deliveries was 1.7231 with a 95\% confidence interval of 0.8989 to 3.3033 with a p-value of 0.1013 . The odds ratio for mothers with five or more previous deliveries was 2.2436 with a $95 \%$ confidence interval of 1.0585 to 4.7552 with a p-value of 0.0350 .

\section{Discussion}

Factors associated with death within $<48$ hours of delivery include increased number of previous maternal pregnancies, increased number of previous deliveries, and decreased birth weight of the neonate. Decreased birth weight was a risk factor separate from gestational age according to the data. Categorical regression analysis indicated that five or more previous maternal deliveries significantly increased the risk of overall neonatal mortality.

Local data can be used to develop and inform interventions for neonatal deaths in this community in sub-Saharan Africa. This would be in concordance with the recent WHO 2014 World Health Assembly agenda [6] which provides new attention toward neonatal deaths on a global level. 
Community education, engagement, and mobilization have been used as strategies toward addressing health problems. There is moderate quality of evidence showing that increasing knowledge and promoting action within the community can increase hospital birth and reduce perinatal and early neonatal mortality [7]. This information could inform the development of a high risk birth screening tool to be used for education and distribution to hospital midwives and antenatal care nurses. Conversations have started with the St. Luke hospital staff as well as traditional birth attendants regarding how best to address the problem of neonatal mortality given their local information.

Implementation of such a tool and the behavior change regarding hospital-based delivery for those at risk may come with challenges. Many of the women at risk have successfully delivered children in out-of-hospital settings. Changing behavior to include hospital delivery for a mother who has had four previous pregnancies will be difficult unless this process is embraced as a community standard. Women in Tanzania were shown to be more likely to use maternal health services with their first-born child than with subsequent pregnancies [8]. Mothers with 1-4 children were more likely to deliver in facility than women with 5 or more children. Women in different parity groups were not statistically different in regard to their distant to nearest facility, female literacy, road conditions, or village poverty. Affordability and past experience with the health system constitute drivers of institutional delivery in this group [8]. All of these issues will be hurdles in tackling the problem of neonatal mortality worldwide.

The contributions of this study are best understood in light of its limitations. The collection of data entirely through retrospective chart review is not without downfalls. The ideal method of data collection used in similar studies includes postmortem interviews with family members and medical providers to establish cause of death. Unfortunately, these sources of data were unavailable for our study. In addition, our data was limited in that it only account for death within the first 48 hours after delivery. The definition of neonatal death is death within 28 days of delivery, but this sort of follow up is simply not possible in the studied setting. However, it is important to remember that previous studies suggest that $25 \%$ to $45 \%$ of all neonatal deaths occur in the first 24 hours of life so our data remains important [9]. Finally, it is important to understand that this data cannot be generalized to the community as a whole as it only provides clinic-based information and thus provides a conservative picture of mortality, as often those most at risk of complication are least likely to seek clinical care.

\section{Conclusion}

This study of data from a rural hospital system suggests that increased risk for neonatal death is significant in mothers with greater than five maternal pregnancies and deliveries. Screening tools to identify women with 5 or more previous pregnancies, women who are at risk of developing labor complications, could result in reduced neonatal death. Identifying these women early in pregnancy using community-based strategies may increase the number of women presenting to a healthcare facility early in labor in order to increase her chance of a healthy birth.

\section{References}

1. Lawn J, Lee AC, Kinney M, Sibley L, Carlo WA, et al. (2009) Two million intrapartum-related stillbirths and neonatal deaths: where, why, and what can be done? Int J Gynaecol Obstet 107: 5-19.

2. UN IGME (2013) Levels and Trends in Child mortality. United Nations Children's Fund, WHO, the World Bank, and the United Nations Population Division. New York, NY, USA.

3. Cousens S, Blencowe H, Stanton C, Chou D, Ahmed S, et al. (2011) National, regional, and worldwide estimates of stillbirth rates in 2009 with trends since 1995: a systematic analysis. The Lancet 377: 1319-1330.

4. Engmann C, Walega P, Aborigo RA, Adongo P, Moyer C, et al. (2012) Stillbirths and early neonatal mortality in rural Northern Ghana. Trop Med Int Health 17: 272-282.

5. Baiden F, Hodgson A, Adjuik M, Adongo P, Ayaga B et al. (2006) Trend and causes of neonatal mortality in the Kassena-Nankana district of northern Ghana, 1995-2002. Trop Med Int Health 4: 532-539.

6. Lawn J (2015) Every Newborn Action Plan. Paper presented at the 2014 World Health Organization, World Health Assembly, Geneva, Switzerland.

7. Lawn J, Shibuya K, Stein C (2005) No cry at birth: global estimates of intrapartum stillbirths and intrapartum-related neonatal deaths. Bulletin of World Health Organization 83: 401-480.

8. Ndao-Brumblay SK, Mbaruku G, Kruk ME (2013) Parity and institutional delivery in rural Tanzania: a multilevel analysis and policy implications. Health Policy and Planning 28: 647-657.

9. Edmond K, Quigley M, Zandoh C, Danso S, Hurt C, et al. (2008) Aetiology of stillbirths and neonatal deaths in rural Ghana: implications for health programming in developing countries. Paediatr Perinat Epidemiol 22: 430-437. 